\title{
CHILEAN LEGENDS: PLATAFORMA EDUCATIVA PARA EL APRENDIZAJE DEL INGLÉS A TRAVÉS DE LEYENDAS DE PUEBLOS ORIGINARIOS
}

\section{CHILEAN LEGENDS: AN EDUCATIVE PLATFORM FOR ENGLISH LEARNING THROUGH LEGENDS OF INDIGENOUS PEOPLE}

\author{
Javiera Andrea Waghorn Mauri ${ }^{1}$; Pamela Karina Miranda Guerrero ${ }^{2}$; Daniela Aguilera \\ Olguín ${ }^{3}$
}

\section{RESUMEN}

Esta investigación analizó los resultados de una experiencia que busca desarrollar las habilidades de la lengua inglesa mediante la metodología basada en tareas y el uso de tecnologías de la información y comunicación (TIC) en un grupo de estudiantes de un segundo medio pertenecientes a una escuela pública de la ciudad de Valparaíso. Se recogieron datos sobre la experiencia a través de pruebas en donde se desarrollaban las habilidades de lectura y auditiva y a través de la observación del trabajo de los educandos. El análisis de los datos género un hallazgo principal: el aprendizaje basado en tareas con herramientas TIC ayudó a los estudiantes a tener un mejor desarrollo de sus habilidades receptivas en el idioma.

Palabras Clave: Metodologías tradicionales, TIC, tareas, habilidades receptivas, L2, motivación.

\section{ABSTRACT}

This research analyzed the results of an experience that seeks to develop the skills of the English language through the task- based methodology and the use of information and communication technologies (ICT) in a group of students of a $10^{\text {th }}$ grade belonging to a public school of the city of Valparaíso. The data about the experience was collected through tests in which reading and listening skills were developed and through observing students work while performing the tasks. The analysis of the data generated one main finding: that learning with tasks and the use of ICT tools helped students to a better development of their receptive skills in the language

Keywords: Traditional methodologies, ICT, Task Based, Receptive skills, L2, motivation.

\section{INTRODUCCIÓN}

Enseñar inglés como lengua extranjera (EFL) es una tarea extenuante en muchos contextos, ya que a pesar de todas las investigaciones realizadas en este campo, hay un cierto grado de insatisfacción cuando observamos la relación entre las estrategias aplicadas en este proceso y la efectividad del dominio de la lengua meta (L2) expresada por los hablantes de los distintos niveles de inglés. Además de la selección de ciertas metodologías tradicionales, las cuales se consideran poco atractivas para el alumnado y cómo Dr. Wendell dice: "La gramática sigue dominando la enseñanza de todas las áreas: en la enseñanza de la formación inicial, en el currículum y, consecuentemente en la enseñanza y aprendizaje del aula." (Citado en Roa, 2011, p. 44). También es posible observar la influencia de una serie de factores que pueden interferir 
en el proceso de aprendizaje adecuado para los estudiantes. Por ejemplo, la ansiedad de los educandos podría interferir en el proceso de aprendizaje de idiomas. Del mismo modo, Aránguiz y Quintanilla en su artículo de retroalimentación correctiva oral en EFL, explican que los estudiantes también se niegan a participar en clases porque tienen miedo de cometer errores debido a razones culturales (Aranguiz \& Quintanilla, 2016, p.105).

Esta es la razón por la cual el gobierno Chileno ha manifestado el deseo de mejorar el sistema educacional a través de la última reforma presentada. Puesto que se han hecho bastantes modificaciones a través de los años, como la incorporación de la ley de Subvención Escolar Preferencial, la cual se refiere a entregar recursos para incorporar nuevas herramientas para la enseñanza, tales como tecnologías de la información y la comunicación (TIC) que aseguran diferentes enfoques a través de tareas (metodología de task-based) para enseñar a los estudiantes aumentando su motivación, creatividad y adquisición de contenidos.

"el uso de las TIC en los centros educativos se impone y sustituye a antiguos usos y recursos. El uso del ordenador y el software educativo como herramienta de investigación, manipulación y expresión tiene una cualidad muy motivadora y atractiva para el alumnado de los distintos niveles educativos.”(Durán, Barrio, \& Herrán, 2007, p. 91).

Tal como se mostró en un estudio realizado en "Florida State University" en una clase de español, donde se estudió la percepción de los estudiantes sobre el uso de la tecnología a través de tareas enfocadas para la adquisición de un segundo idioma, se confirmó que las TIC son beneficiarias, ya que la mayoría de los estudiantes en este estudio confirmaron haber estado más interesados en las tareas de la clase y haber aumentado su adquisición en la L2 “(...) students agreed that the computer lab made the course more interesting, and slightly more than half said that they would take another technology-enhanced class in Spanish (...)" and "(...) two-thirds or more of the students reported that their listening and reading skills, as well as their cultural knowledge, had improved (...)" (Stepp-Greany, 2002, p.170).

Es por esto que nuestra propuesta metodológica es la implementación de Task Based language learning (TBLT) a través de tecnologías de la información y comunicación (TIC) para la adquisición y desarrollo de habilidades receptivas en una L2 y para el aumento del interés y motivación estudiantil hacia el aprendizaje del inglés.

Nuestro estudio fue dirigido, en un segundo medio de un Liceo público en la ciudad de Valparaíso en Chile, en donde el desarrollo del inglés es deficiente, ya que los estudiantes no se sienten interesados en su aprendizaje o en el desarrollo de habilidades en la L2. 
El objetivo de esta investigación es demostrar si realmente el uso de metodología Task-based con herramientas tecnológicas tienen un efecto positivo en el aumento de interés por parte de los educandos hacia la adquisición de la L2.

\section{FUNDAMENTO TEÓRICO}

El aprendizaje de idiomas implica un cierto grado de dominio de las cuatro habilidades básicas que permiten a los estudiantes comprender y producir la lengua meta. Estas cuatro habilidades comunicativas son leer, escuchar, hablar y escribir; las cuales son necesarias para el desarrollo de una comunicación efectiva en cualquier idioma (Osbor, Osborn \& Osborn, 2008, p.1036). Mientras que leer y escuchar se consideran habilidades receptivas, las otras son habilidades productivas.

En el sistema educacional Chileno se muestra deficiente en cuanto al desarrollo de habilidades dentro del idioma inglés tal como afirmó el ministerio de educación, “(...) al finalizar la Enseñanza Media, un porcentaje menor de alumnos alcanza el nivel de inglés 'Autónomo' por lo que los estudiantes estarían muy lejos de dominar el inglés necesario para desempeñarse efectiva y eficientemente en el ámbito laboral y educacional" (Citado en Blázquez \& Tagle, 2012).

Esto sucede ya que los estudiantes de lenguas extranjeras generalmente "tienen oportunidades limitadas para hablar inglés fuera del aula" (Zhang, 2009 citado en Kong, 2011, p.74) y también una exposición limitada a relacionarse con angloparlantes.

Otra dificultad es el uso de metodologías tradicionales, por ejemplo el método de traducción de la gramática el cual ha sido ampliamente considerado como el enfoque oficial de enseñanza de lenguas extranjeras durante los siglos XVIII y XIX. En el cual la lengua meta se traduce a la madre y su principal metodología tiene que ver con la adquisición de reglas gramaticales de la L2 y el uso posterior al traducir literatura y oraciones de L2 a L1 (Kong, 2011, p.76). Por otro lado, la adopción masiva de este método comenzó a cuestionarse a fines del siglo XX debido a algunas asociaciones negativas que evitan que la adquisición de la lengua sea lo más completa posible (Kong, 2011, p.76).

Al tener en cuenta las claras limitaciones del método de traducción gramatical, nos parece adecuado sugerir una implementación progresiva del método task-based con el uso de las TIC, los cuales podrían demostrar su utilidad cuando se implementa en la búsqueda del aprendizaje de habilidades lingüísticas múltiples y como existen variados tipos de estructuras de esta metodología, las cuales difieren de un autor a otro, seleccionamos la estructura propuesta por Jane Willis, donde el autor establece una primera etapa denominada la fase previa 
a la tarea, una etapa de desarrollo conocida como Taskcycle y finalmente la etapa de Focus on form, en las cuales las dos primeras etapas se basan en la norma sociolingüística y aseguran una transición suave y natural de la interacción privada a la pública, así pues la última fase establece oportunidades de practicar los parámetros formales del lenguaje (Willis, 1996, p.4).

Es por todo esto que se creó una plataforma virtual con leyendas tradicionales chilenas cuyo objetivo es desarrollar las habilidades en la L2 e incrementar la motivación de los estudiantes hacia su aprendizaje.

En el diseño de la propuesta metodológica se consideraron los siguientes objetivos curriculares vigentes:

Tabla: Comparación de los objetivos del Ministerio de Educación de Chile y de la aplicación. Fuente: Ministerio de Educación y Chilean Legends

\begin{tabular}{|c|c|}
\hline $\begin{array}{c}\text { Objetivos de Aprendizaje del Ministerio } \\
\text { de Educación }\end{array}$ & $\begin{array}{l}\text { Objetivos de Aprendizaje del Ministerio } \\
\text { de Educación }\end{array}$ \\
\hline $\begin{array}{l}\text { Demostrar comprensión de ideas generales e } \\
\text { información explícita en textos adaptados y } \\
\text { auténticos simples, en formato impreso o digital, } \\
\text { acerca de temas variados (como temas de interés } \\
\text { de los y las estudiantes, temas de otras } \\
\text { asignaturas, del contexto inmediato, de } \\
\text { actualidad e interés global, de otras culturas y } \\
\text { algunos temas menos conocidos) y que } \\
\text { contienen las funciones del año. }\end{array}$ & $\begin{array}{l}\text { OA9 Reconocer elementos relacionados con la } \\
\text { cultura inca a través de un texto interactivo, } \\
\text { desarrollando el respeto por otras culturas. }\end{array}$ \\
\hline $\begin{array}{l}\text { Demostrar comprensión de ideas generales e } \\
\text { información explícita en textos orales adaptados } \\
\text { y auténticos simples, literarios y no literarios, en } \\
\text { diversos formatos audiovisuales (como } \\
\text { exposiciones orales, conversaciones, } \\
\text { descripciones, instrucciones y procedimientos, } \\
\text { discursos y debates breves y simples, avisos } \\
\text { publicitarios, entrevistas, noticieros, anécdotas, } \\
\text { narraciones, canciones), acerca de temas } \\
\text { variados (experiencias personales, temas de } \\
\text { otras asignaturas, del contexto inmediato, de } \\
\text { actualidad e interés global o de otras culturas) y } \\
\text { que contienen las funciones del año. }\end{array}$ & $\begin{array}{l}\text { OA1 Reconocer elementos relacionados con } \\
\text { prácticas de la cultura inca, a través de textos } \\
\text { escuchados de manera de audiovisual, } \\
\text { desarrollando el respeto por otras culturas. }\end{array}$ \\
\hline $\begin{array}{l}\text { Elaborar una leyenda considerando elementos de } \\
\text { la cultura inca, a partir del texto y material } \\
\text { audiovisual, teniendo en cuenta frases, palabra o } \\
\text { expresiones para desarrollar el interés por otra } \\
\text { cultura. }\end{array}$ & $\begin{array}{l}\text { OA13 Escribir historias e información relevante, } \\
\text { usando diversos recursos multimodales en forma } \\
\text { creativa y efectiva que refuercen el mensaje en } \\
\text { textos variados, acerca de temas como: } \\
\text { - Experiencias personales. } \\
\text { - Contenidos interdisciplinarios. } \\
\text { - Aspectos de interés global. } \\
\text { - Cultura de otros países. }\end{array}$ \\
\hline
\end{tabular}




\begin{tabular}{|l|l|}
\hline $\begin{array}{l}\text { Discutir sobre la cultura Inca a través de } \\
\text { preguntas que orientan la reflexión grupal de } \\
\text { manera respetuosa. }\end{array}$ & $\begin{array}{l}\text { OA5 Presentar información en forma oral, } \\
\text { usando recursos multimodales que refuercen el } \\
\text { mensaje en forma creativa, acerca de temas } \\
\text { variados (como experiencias personales, temas } \\
\text { de otras asignaturas, otras culturas, problemas } \\
\text { globales y textos leídos o escuchados), } \\
\text { demostrando: } \\
\text { Conocimiento del contenido } \\
\text { coherencia al organizar ideas. } \\
\text { Uso apropiado de las funciones del } \\
\text { lenguaje yocabulario del nivel. } \\
\text { Tener conciencia de audiencia, contexto } \\
\text { y propósito. }\end{array}$ \\
\hline
\end{tabular}

Para crear este recurso se utilizaron las siguientes herramientas:

- Wix.com: Herramienta que permite crear páginas web, la cual se utilizó como repositorio de aprendizaje

- H5p.org: esta herramienta fue utilizada para la creación de las actividades interactivas con uso de TIC en donde los estudiantes pueden practicar las habilidades en la L2.

- Chileprecolombino.cl: esta página web fue utilizada para obtener las ilustraciones de las culturas precolombinas chilenas para la representación de cada una de las leyendas.

Este recurso fue creado bajo la metodología TBLT el cual está empezando a influir en el uso de estrategias de aprendizaje, no solo en la enseñanza de EFL. En la instrucción de taskbased, el trabajo de pares o en grupo a menudo se utiliza para aumentar la interacción y la colaboración de los educandos. También los estudiantes participan en tareas comunicativas en inglés. Estas se definen como actividades que pueden ser trabajadas de forma independiente o como unidades, las cuales requieran comprender, producir, manipular o interactuar en un lenguaje auténtico, la atención de esta metodología se centra principalmente al significado más que la forma (Carless, 2007, p. 597).

Otra característica fundamental de este método basado en tareas tiene que ver con la implementación de objetivos observables que los alumnos notan claramente durante el desarrollo de las mismas. Este aspecto transforma las metas en tareas concretas y factibles que promueven la adquisición del lenguaje utilizando contextos familiares que apoyan el desarrollo del habla (Correa, Lara, Pino \& Vera, 2017, p.146). 
Es por esto que el uso de la metodología Task based language learning se muestra beneficiosa para la adquisición de una L2 e implementada con el uso de las TIC se vuelve una herramienta motivante para los estudiantes "the use of ITs implemented with the use of TBLT may assure an increase in students' motivation towards English learning, particularly if taskand-technology integrations are properly motivated by TBLT theory (...) they can raise students' motivation to take risks and be creative while using language to make meaning (...)" (González-Lloret y Ortega, 2014, p. 4). Tal como Roblyer y Edwards afirmaron: Las metodologías basadas en el uso de herramientas TIC se muestran poderosas a la hora de entregar estrategias para incrementar la motivación en los educandos, las culeas pueden ser utilizadas de manera individual o en conjunto (citado en Tuan \& Doan, 2010, p. 65).

Using ICT in a creative students centered way can mean to integrate the use of unique tasks, such as e-creation tools that promote interaction between students and enhance the development of the 4 skills (Erben, Ban, y Castañeda, 2009, p112) y los profesores de una segunda lengua (L2) deben estar conscientes para comprometer a sus estudiantes en su proceso de aprendizaje “(...) Por lo tanto se considera importante que cualquier profesional interesado en el aprendizaje y enseñanza de una segunda lengua en el siglo XXI debería investigar el uso de las metodologías innovadas, como lo es task-based, con la implementación de las TIC, ya que los educandos se pueden comprometer en tareas en las cuales están utilizando la L2 de manera autentica lo que provocan de manera efectiva la adquisición de la lengua practicada (Chapelle, 2001, p.2).

\section{METODOLOGÍA}

Esta investigación fue de tipo cuantitativa y experimental ya que los resultados fueron obtenidos a través de estadísticas numéricas con la aplicación de pruebas controladas.

\section{PARTICIPANTES:}

El estudio fue conducido en un Liceo público mixto en la ciudad de Valparaíso, Chile, donde los estudiantes tenían un nivel básico y presentaban dificultades al momento de entender y expresar el idioma inglés. Los participantes fueron 15 estudiantes para desarrollar la habilidad de comprensión de lectura (Reading) y 11 estudiantes para desarrollar la habilidad de comprensión auditiva (Listening).

\section{INSTRUMENTOS}

Los instrumento utilizados para la recolección de datos fueron dos pruebas sistemáticas para desarrollar la habilidad auditiva y la habilidad de comprensión lectora (ilustración 1 y 2) 
MAURI et al

en las cuales los estudiantes tuvieron que responder rellenando espacios en blanco de un texto previamente presentado a través de una página web (http://bit.ly/chileanlegends) y contestando preguntas de selección múltiple mientras visualizaban un video a través de una plataforma interactiva virtual conectada con su realidad, la cual son los pueblos originarios Chilenos.

Ilustración 1 y 2: Guías de aprendizaje. Fuente propia.
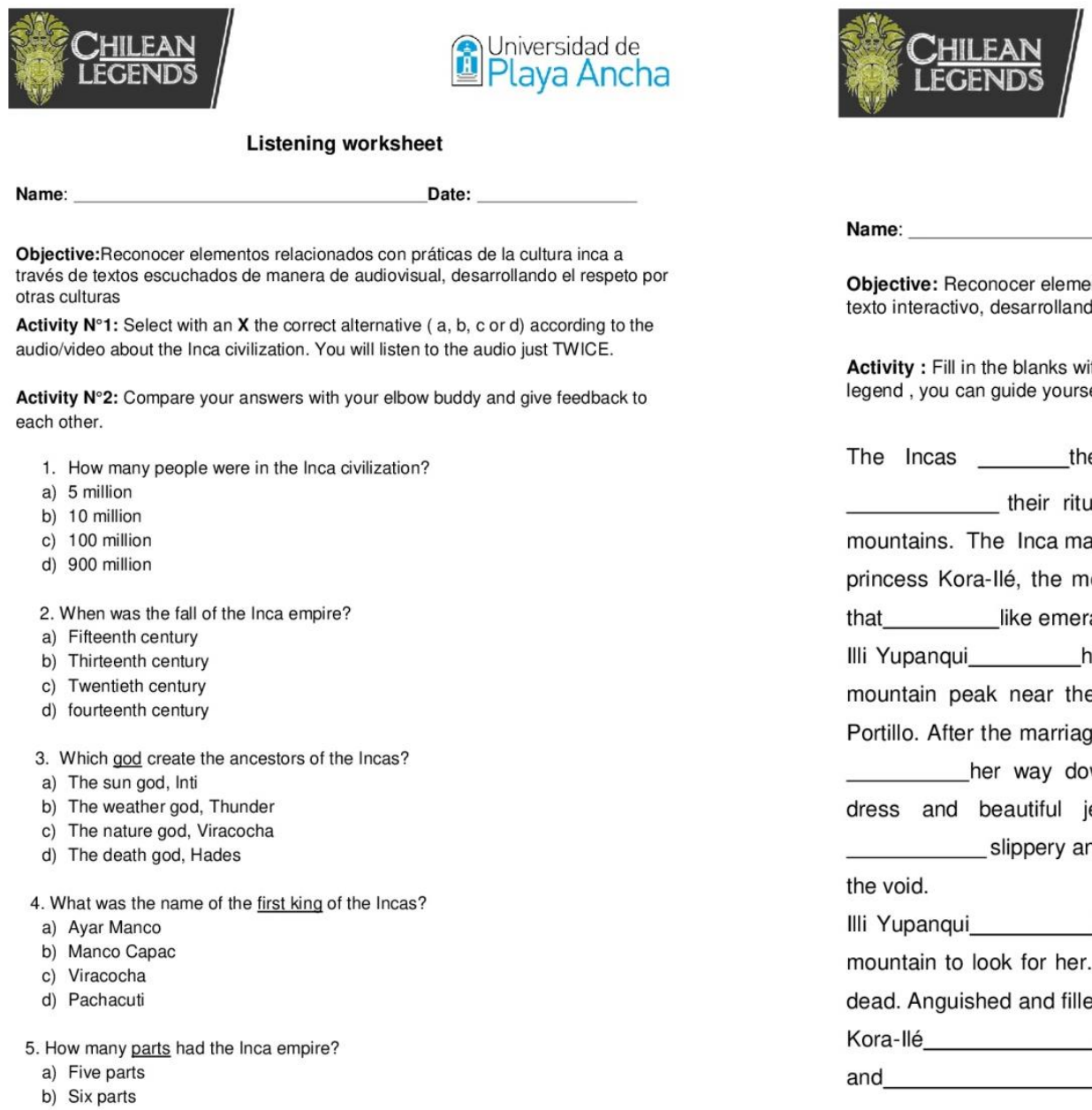
Aniversidad de i. Playa Ancha

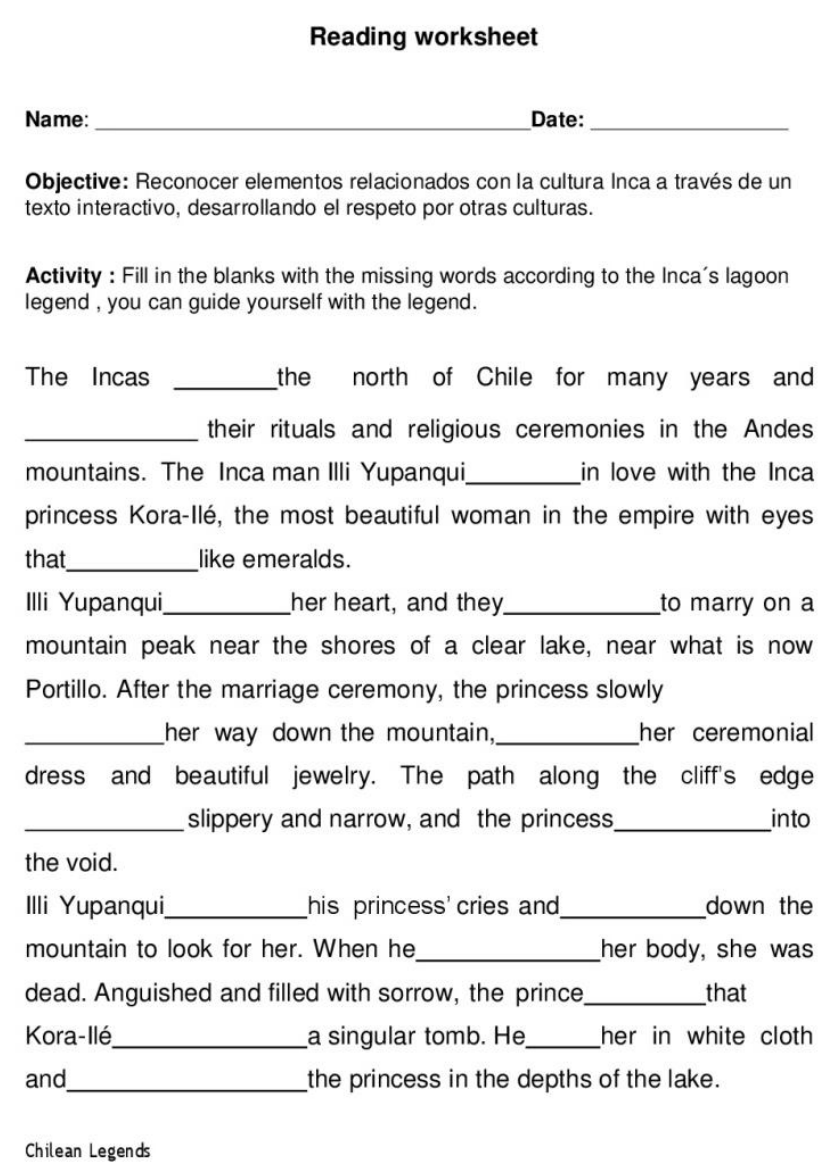

\section{PROCEDIMIENTO:}

El procedimiento llevado a cabo fue observar el contexto de estos estudiantes y sus falencias hacia el aprendizaje de un segundo idioma para luego crear una estrategia con el objetivo de aumentar su motivación hacia su aprendizaje en la L2 y mejorar el desarrollo de las habilidades receptivas.

El primer paso fue dirigir a los estudiantes a la sala de computación en el establecimiento educacional, para luego presentarles los objetivos esperados de la clase, los cuales fueron: 
Habilidad de comprensión lectora (Reading): Comprender texto relacionado a la cultura precolombina Chilena para identificar vocabulario específico para así completar una guía de aprendizaje promoviendo el respeto.

Habilidad de comprensión auditiva (Listening): Comprender un video relacionado con la cultura inca para identificar y relacionar información específica para así completar una guía de aprendizaje promoviendo la diversidad y el respeto.

Luego los estudiantes exploraron la página virtual de Chilean Legends para interiorizar su uso mientras se explicaban las actividades para luego proceder a completar las tareas de reading y listening junto al apoyo y monitoreo de la profesora.

Finalmente se evaluó el logro de los objetivos con los estudiantes a través de sus percepciones con respecto al trabajo realizado.

\section{RESULTADOS Y DISCUSIÓN}

En nuestra primera intervención con la plataforma Chilean Legends (bit.ly/chileanlegends) realizada en un segundo medio del Liceo Técnico en la ciudad de Valparaíso en Chile, observamos que la utilización de una plataforma virtual de aprendizaje permite que los estudiantes trabajen de manera autónoma o grupal motivados en el aprendizaje del inglés.

Los resultados que se obtuvieron al aplicar el diseño de nuestra aplicación fueron los siguientes:

Tabla 1: resultados generales de las guías de aprendizaje. Fuente propia.

\begin{tabular}{|c|c|c|c|}
\hline \multicolumn{3}{|c|}{ Resultados Generales } \\
\hline \multicolumn{1}{|c|}{ Habilidades } & \multicolumn{1}{|c|}{ Logro } & Alumnos & Porcentaje \\
\hline \multirow{2}{*}{ Reading } & Sobre $60 \%$ & 12 & $80 \%$ \\
\cline { 2 - 4 } & Bajo $60 \%$ & 3 & $20 \%$ \\
\hline \multirow{2}{*}{ Listening } & Sobre $60 \%$ & 7 & $63,6 \%$ \\
\cline { 2 - 4 } & Bajo $60 \%$ & 4 & $36,4 \%$ \\
\hline
\end{tabular}


Tabla 2: detalle de resultados sobre la habilidad de lectura (reading) Fuente propia.

\begin{tabular}{|l|l|l|l|l|l|}
\hline \multicolumn{7}{|c|}{ Detalle de Resultados : Habilidad Reading } \\
\hline $\begin{array}{l}\text { Preguntas } \\
\text { Correctas }\end{array}$ & 20 & 19 & 18 & 3 & 0 \\
\hline Alumnos & 7 & 3 & 2 & 1 & 2 \\
\hline Porcentajes & $47 \%$ & $20 \%$ & $13 \%$ & $6,6 \%$ & $13,3 \%$ \\
\hline
\end{tabular}

Tabla 3: detalle de resultados de la habilidad auditiva (listening) Fuente propia.

\begin{tabular}{|l|l|l|l|l|l|l|l|}
\hline \multicolumn{7}{|c|}{ Detalle de Resultados : Habilidad Listening } \\
\hline $\begin{array}{l}\text { Preguntas } \\
\text { Correctas }\end{array}$ & 10 & 9 & 8 & 7 & 6 & 3 & 1 \\
\hline Alumnos & 0 & 3 & 2 & 1 & 1 & 1 & 3 \\
\hline $\begin{array}{l}\text { Porcentaje } \\
\text { S }\end{array}$ & $0 \%$ & $\begin{array}{l}27,3 \\
\%\end{array}$ & $\begin{array}{l}18,1 \\
\%\end{array}$ & $9,1 \%$ & $9,1 \%$ & $\begin{array}{l}9,1 \\
\%\end{array}$ & $\begin{array}{l}27,3 \\
\%\end{array}$ \\
\hline
\end{tabular}

\section{CONCLUSIÓN}

Evidenciamos que nuestra experiencia fue positiva tanto para nosotras como para los educandos, quienes reflejaron un trabajo autónomo.

Sin duda, el mayor aporte que entrega nuestra aplicación y sus resultados es que abre la posibilidad de investigar acerca de si se logra un aprendizaje significativo del inglés a partir de un material visto desde una cultura angloparlante o desde un material adaptado a la realidad Chilena.

\section{REFERENCIAS}

Aranguiz M. F., \& Espinoza, A. Q. (2016). Oral Corrective Feedback Strategies in EFL. A Pilot Study in Chilean Classrooms. Elia, (16), 103-132. doi:10.12795/elia.2016.i16.05

Blázquez, F., \& Tagle, T. (2012). Formación docente: un estudio de las creencias de alumnos y profesores sobre el proceso de enseñanza y aprendizaje del inglés. 
Carless, D. (2007). The suitability of task-based approaches for secondary schools: Perspectives from Hong Kong. System, 35(4), 595-608. doi:10.1016/j.system.2007.09.003 Chile continues to push for improved English proficiency. (2016, April 29). Retrieved November 24, 2017.http://monitor.icef.com/2016/04/chile-continues-to-push-for-improvedenglish-proficiency/

Chang, S. (2011, 05). A Contrastive Study of Grammar Translation Method and Communicative Approach in Teaching English Grammar.English Language Teaching, 4(2), 13. doi:10.5539/elt.v4n2p13.

Chapelle, C. A. (2001). Computer applications in second language acquisition. Cambridge University Press.

Correa, R., Lara, E., Pino, P., \& Vera, T. (2017, 02). "Relationship Between Group SeatingArrangement in the Classroom and Student Participation in Speaking Activities in EFLClasses at a Secondary School in Chile". Folios, 45(1), 145-158. doi:10.17227/01234870.45folios145.158.

Durán, A. F., Barrio, J. F. B., \& Herrán, A. (2007). Recursos informáticos para la enseñanzaaprendizaje del inglés en educación infantil y primer ciclo de primaria: una investigación en el aula. Porta Linguarum: Revista Internacional de Didáctica de Las Lenguas Extranjeras, (7), 7. Retrieved from https://dialnet.unirioja.es/servlet/articulo?codigo=2371719

EDUCACIÓN, M. D. (2008, March 07). DTO-81 14-JUN-2004 MINISTERIO DE EDUCACIÓN. Retrieved fromhttps://www.leychile.cl/Navegar?idNorma=226283 Erben, T., Ban, R., \& Castañeda, M. E. (2009). Teaching English language learners through technology. New York: Routledge.

González-Lloret, M., \& Ortega, L. (2014). Technology-mediated TBLT: Researching Technology and Tasks. John Benjamins Publishing Company. p.1-5

Kong, N. (2011, 02). Establishing a Comprehensive English Teaching Pattern Combining the Communicative Teaching Method and the Grammar-Translation Method. English Language Teaching,4(1), 76. doi:10.5539/elt.v4n1p76

Osborn, S., Osborn, M., \& Osborn, R. (2008). Public speaking guidebook. Pearson/Allyn and Bacon.

Roa G. (2011). Aproximación al sistema de creencias pedagógicas del profesor de inglés en la enseñanza de la habilidad de expresión oral del idioma. El caso de un colegio bilingüe. p. 41-73.

Savignon, S. J. (2017, 03). Communicative Competence. The TESOL Encyclopedia of English Language Teaching, 1-7. doi:10.1002/9781118784235.eelt0047

Stepp-Greany J.(2002). Perceptionson language learning in a technological environment: Implications for the new millennium. Language Learning \& Technology Vol. 6, Num. 1 (p. 165-180) doi: 10125/25148 
Sierra, F.C., \& Alcalá, U.D. (2007). Foreign Language Teaching Methods: Some Issuesand New Moves.

Tuan, L. T., \& Doan, N. T. M. (2010). Teaching English grammar through games. Studies in Literature and Language, 1(7), 61. Retrieved from http://search.proquest.com/openview/54163147011e28eb87f9322843a77705/1?pqorigsite $=$ gscholar \&cbl $=436445$

Willis, J. (1996). A Framework for Task-Based Learning. Harlow: Longman.

Yilorm, Y. (2016). Proceso de enseñanza aprendizaje de la lengua inglesa en escuelas públicas chilenas: ¿Producción o reproducción? Estudios Pedagógicos (Valdivia), 42(Especial), 103-116. doi:10.4067/s0718-07052016000300009 